\title{
Correlation of echocardiographic findings of pulmonary hypertension with six-minute walk test and plasma pro b-type natriuretic peptide level in systemic lupus erythematous
}

\author{
Leila Ghofraniha ${ }^{1}$, Zahra Mirfeizi² ${ }^{2}$ Fatemeh Seyyedi Khabbaz ${ }^{2}$, Farveh Vakilian ${ }^{3}$, Saeed Eslami ${ }^{4,5}$
}

${ }^{1}$ Pulmonologist, Lung Disease Research Center, School of Medicine, Mashhad University of Medicine Sciences, Mashhad, Iran

${ }^{2}$ MD, Associate Professor of Rheumatology, Rheumatic Diseases Research Center, School of Medicine, Mashhad University of Medical Sciences, Mashhad, Iran

${ }^{3}$ Internist, Rheumatic Diseases Research Center, Preventive Atherosclerotic Research Center, Cardiology Department, Imam Reza Hospital, Mashhad University of Medical Sciences, Mashhad, Iran

${ }^{4}$ Internal Medicine, Pharmaceutical Research Center, School of Pharmacy, Mashhad University of Medical Sciences, Mashhad, Iran

${ }^{5}$ Department of Medical Informatics, School of Medicine, Mashhad University of Medical Sciences, Mashhad, Iran

Type of article: Original

\begin{abstract}
Introduction: Pulmonary arterial hypertension (PAH) is an increasingly recognized complication of systemic lupus erythematous (SLE), which may remain undiagnosed if asymptomatic.

Objective: This study aimed to determine the correlation between echocardiographic findings of PAH and sixminute walk test (6WMT) and serum pro b-type natriuretic peptide (proBNP) level in patients with SLE.

Methods: This cross-sectional study was performed on 50 SLE patients selected from patients referring to the outpatient's department of the Rheumatology Clinic at Imam Reza Hospital in Mashhad, Iran, from July 2013 through September 2014, using resting transthoracic echocardiography to estimate systolic pulmonary artery pressure (sPAP). Variables were summarized as counts and/or percentages or as mean $\pm \mathrm{SD}$. Inter-group comparisons were made performing two-tailed Fisher's exact test or Mann-Whitney U test, using SPSS 22.

Results: In general, five out of fifty patients were diagnosed to have PAH with sPAP $>30 \mathrm{mmHg}$ (range: $31-40$ $\mathrm{mmHg}$ ) based on echocardiographic findings. Spirometric parameters did not show any differences between the two groups $(\mathrm{p}>0.05)$, while the difference in total distance walked during six minutes and serum proBNP level between SLE patients with and without PAH was significant $(\mathrm{P}<0.05)$. A high correlation was found between PAP and serum proBNP level, but not between PAP and the distance walked during six-minutes in SLE patients. Conclusion: The point prevalence of PAH in SLE patients was 10\%; the significant correlation between PAP and serum proBNP level suggests that it can be used as a valuable marker for early diagnosis of asymptomatic pulmonary hypertension in patients with SLE.

Keywords: Systemic lupus erythematosus, Pulmonary arterial hypertension, Six-minute walk test, Serum proBNP levels, Transthoracic echocardiography
\end{abstract}

\section{Introduction}

Systemic lupus erythematous (SLE) is a well-recognized autoimmune multisystem disorder with frequent respiratory and cardiac manifestations (1) that can lead to high rates of morbidity and mortality $(2,3)$. To improve prognosis of pulmonary hypertension, new treatment methods have been proposed $(4,5)$. A multitude of studies were conducted on patients with idiopathic pulmonary hypertension (PAH) and connective tissue disease patients with systemic sclerosis (SSc) (4-6). Approximately $0.5-43 \%$ of the cases of PAH are assumed to be associated with SLE (7-10). These results were reported in retrospective studies involving large numbers of SLE patients over a 5

\section{Corresponding author:}

Associate Professor Dr. Zahra Mirfeizi, Rheumatic Diseases Research Center (RDRC), Mashhad University of Medical Sciences, Mashhad, Iran. Tel: +985138012753, Fax:+985138401036, Email: Mirfeiziz@mums.ac.ir Received: August 29, 2016, Accepted: March 14, 2017, Published: August 2017 iThenticate screening: January 23, 2017, English editing: July 20, 2017, Quality control: August 12, 2017 (C) 2017 The Authors. This is an open access article under the terms of the Creative Commons Attribution-NonCommercialNoDerivs License, which permits use and distribution in any medium, provided the original work is properly cited, the use is non-commercial and no modifications or adaptations are made. 
to10-year period or cross-sectional studies on limited groups of patients (11). The wide variability in these prevalence estimates reflect the differences in PAH definitions, population groups, sample sizes, and the applied diagnostic methods (11). The nonspecific nature of symptoms associated with PAH such as dyspnea, fatigue, palpitation, and syncope can result in delayed diagnosis of PAH in SLE patients, indicating the need for appropriate screening methods to identify PAH (11). Although the gold standard test for diagnosis of PAH is right heart catheterization (RHC), its invasiveness and cost make it an unsuitable screening tool (12). Transthoracic Doppler echocardiography, however, is considered a safe, sensitive, and specific tool for screening PAH and assessing its severity in SSc patients $(13,14)$. In the current study, we aimed to prospectively determine the prevalence of PAH in SLE patients using echocardiography and assess the value of pulmonary function tests, 6MWT, and proBNP level as screening tests for PAH. We also determined the correlation of six-minute walk test (6WMT) and proBNP level with pulmonary arterial pressure as estimated by echocardiography in lupus patients.

\section{Material and Methods}

\subsection{Study design and setting}

This cross-sectional study was carried out on fifty SLE patients, selected from patients referring to the outpatient's department of the Rheumatology Clinic at Imam Reza Hospital in Mashhad, Iran, from July 2013 through September 2014. The sampling method was convenient and the cases were among the referrals to our teaching hospital.

\subsection{Selection criteria}

The inclusion criteria comprised of patients meeting the 1982 revised criteria of the American College of Rheumatology (ACR) for diagnosis of SLE (15), who did not present evidence of other connective tissue diseases. The exclusion criteria included 1) left heart disease, 2) consumption of drugs such as fenfluramine and aminorex, 3) HIV infection, 4) portal hypertension, 5) chronic hemolytic, 6) anemia, 7) pregnancy, 8) significant renal impairment (creatinine $\geq 150 \mathrm{mmol} / \mathrm{L}$ ), 9) history of unstable angina or a myocardial infarction during the previous month, 10) resting tachycardia (heart rate $\geq 120$ beats/min), 11) uncontrolled hypertension $\geq 180 / 100 \mathrm{mmHg}, 12$ ) history of cardiac arrhythmia, and 13) arthritis and other musculoskeletal diseases.

\subsection{Data sources and measurements}

\subsubsection{Questionnaire}

The patients completed a questionnaire including the patients' demographic data, medication history, and previous medical documents, and underwent transthoracic echocardiography to identify evidence of PAH. Blood samples were obtained for evaluation of serum proBNP level; then, a pulmonary function test (spirometric test) was run to investigate subclinical pulmonary involvement, and 6 MWT was carried out to assess exercise capacity. A questionnaire was completed based on the patients' demographics and previous medical documents including previous history of Raynaud's phenomenon, previous history of thrombosis, certain autoantibody positivity at any time during the course of the disease, and medication history.

\subsubsection{Echocardiography}

Two-dimensional, M-mode color Doppler echocardiography was performed for all the patients at rest using a transthoracic echocardiography device by an expert cardiologist, who had no knowledge of the patients' past medical history. Parasternal long and short axis, four-chamber projections, and evidence of pulmonary hypertension (PH) including systolic PAP, tricuspid annular plane systolic excursion (TAPSE), left ventricular ejection fraction (EF), and pericardial effusion were recorded in all the patients. The World Health Organization defines PAH as mean $\mathrm{PAP}>25 \mathrm{mmHg}$ at rest and $>30 \mathrm{mmHg}$ with exercise, in the presence of normal pulmonary capillary wedge pressure as measured by RHC (11). Similar to former studies, the patients with sPAP of higher than $30 \mathrm{mmHg}$ at rest were diagnosed with PHA, using echocardiography as a screening tool for PH (5).

\subsubsection{Blood sampling and assay}

An aliquot of the serum sample was collected and stored in gel separator tubes on the same day. Samples were transferred to the Chemical Pathology Department in serum gel tubes at room temperature, where they were centrifuged and stored at $-20 \mathrm{oC}$ for later analysis. Thereafter, the electrochemiluminescence immunoassay was performed for quantitative in-vitro determination of NT-proBNP in human serum. According to the manufacturer of the assay, the analytical range for NT-proBNP assay is 5-35,000 pg/ml and the expected NT-proBNP values (95th percentile) for different age groups and each gender are summarized in Table 1. The aforementioned NT-proBNP values adjusted for age and gender were applied to classify our study patients with and without high proBNP concentrations. 


\subsubsection{Spirometry}

Spirometry was performed according to the standards of the American Thoracic Society at the time of examination, by one technician (16). To ascertain repeatability of the test, spirograms were obtained until three admissible forced vital capacity (FVC) maneuvers were conducted (17). We employed Chest Compression System to gauge FVC, and forced expiratory volume in 1 second (FEV1). The observed values for each individual were compared with those predicted for age, gender, and height. Afterwards, we reported the outcomes as the percentage of predicted values. According to ATS standard, when pulmonary function parameters were below $80 \%$ of the predicted value, they were considered abnormal (16). Obstructive pattern was characterized by values below $70 \%$ of FEV1/FVC ratio and less than $80 \%$ of predicted FEV1; further, the restrictive pattern was characterized by FVC values less than $80 \%$ of predicted normal or elevated FEV1/FVC ratio (18). With the subjects in sitting position, measurements were performed without bronchodilator administration.

\subsubsection{Six-minute walk test}

The 6MWT was performed for all the patients using a standardized protocol in accordance with the American Thoracic Society guidelines to estimate exercise capacity (19). This test has good reproducibility and is easy to administer, well-tolerated by patients with respiratory derangements, and is a better reflection of patients' daily activities compared to the shuttle walk test. To perform the 6 MWT, a $15-\mathrm{m}$ walking course was prepared in a corridor with turnaround points marked with bright color tape at 0 and $15 \mathrm{~m}$. Every $5 \mathrm{~m}$ was marked with white tape. In case of fatigue, the patients could sit on chairs provided alongside the corridor. All the participants were asked to wear comfortable clothing, footwear, and walking aids. Before conducting the test, the modified Borg scale for scoring dyspnea and fatigue was used to record the scores at baseline and post-exercise stage, and a pulse oximeter and heart rate monitor were attached to the patients. The subjects were notified that the objective of the study was to walk as far as possible during six minutes and that they were allowed to slowdown, stop and rest (if required), and resume walking. The countdown timer, which was set to six minutes, was started with the patients commencing the test. We informed the participants of the remaining time every minute. We recorded the completed laps and the distance walked in the last partial lap. At the end of the test, Borg dyspnea and fatigue scores were re-recorded, and we made sure that none of the patients had any problems (20). Borg scale is a tool for describing the intensity of perceived dyspnea and fatigue on a scale of zero to ten. At this stage of the study, we recorded the total distance walked (meters) during six minutes, pre- and post-exercise Borg dyspnea and fatigue scores, heart rate (rate/min), and oxygen saturation (\%).

\subsection{Ethical considerations}

The study protocol was approved by the regional Ethics Committee of Mashhad University of Medical Sciences, and written informed consent was obtained from all the subjects for all testing procedures before entering the study.

\subsection{Statistical analysis}

Variables were summarized as counts and/or percentages or as mean \pm SD. Inter-group comparisons were made performing two-tailed Fisher's exact test or Mann-Whitney U test, using IBMC SPSS $\odot$ Statistics version 22 (IBM $\odot$ Corp., Armonk, NY, USA). In spite of being aware of limited significance due to small sample size, correlations between variables were assessed using Pearson product-moment correlation coefficient. A p-value less than 0.05 was considered statistically significant.

Table 1. NT-proBNP values (95th percentile) in different age groups and gender

\begin{tabular}{|l|l|l|}
\hline \multirow{2}{*}{ Age (year) } & \multicolumn{2}{|l|}{$95^{\text {th }}$ percentile NT-proBNP value } \\
\cline { 2 - 3 } & Women & Men \\
\hline$<50$ & $125 \mathrm{pg} / \mathrm{ml}$ & $64 \mathrm{pg} / \mathrm{ml}$ \\
\hline $50-50$ & $186 \mathrm{pg} / \mathrm{ml}$ & $135 \mathrm{pg} / \mathrm{ml}$ \\
\hline$>60$ & $204 \mathrm{pg} / \mathrm{ml}$ & $194 \mathrm{pg} / \mathrm{ml}$ \\
\hline
\end{tabular}

\section{Results}

This study was carried out on 50 SLE patients meeting the updated ACR classification criteria for SLE. Patients with SLE were classified into two groups. Group I included five SLE patients with PAH, and group II comprised of 45 SLE patients without PAH. Demographic data, clinical and paraclinical features, the results of echocardiography, spirometry, serum proBNP level, and 6MWT, as well as the comparison between the two groups are summarized in Table 2. The study groups comprised of 50 SLE patients, 48 (94\%) of whom were females. The mean age of the participants was 29 years (age range: 21 to 48 years) and mean disease duration was four years (range: 1 to 17 years). All the patients were non-smokers. Comparison of lupus patients with and without PAH showed no 
significant differences with respect to gender, age distribution, body mass index (BMI), and disease duration $(\mathrm{p}>0.05)$. Past medical history of the patients revealed that none of them had chronic respiratory symptoms, but Raynaud's phenomenon occurred in $30 \%$ of the patients and only one patient $(2 \%)$ had a clinical clotting episode in the past. Twelve patients (24\%) had previously undergone immunosuppressive therapy for severe organ involvement other than the lung. The frequency of Reynaud's phenomenon, previous history of thrombosis, and prior therapy with immunosuppressive drugs showed no significant differences between the two groups of SLE patients ( $\mathrm{p}>0.05)$. Anti-U1-ribonucleoprotein (anti-U1RNP), rheumatic factor, and anti-phospholipid antibody were positive in $17(34 \%), 7(14 \%)$, and $11(22 \%)$ patients, respectively. SLE patients with PAH did not show a significantly higher number of cases with positive auto-antibodies such as RF, antiU1RNP, and anti-phospholipid antibodies, compared to SLE patients without PAH ( $>0.05)$. According to echocardiographic findings, all the patients $(100 \%)$ had normal left ventricular function and none of them had valvular heart disease or pericardial effusion. Five out of fifty $(10 \%)$ patients had $\mathrm{sPAP}>30 \mathrm{mmHg}$ (pulmonary hypertension) on echocardiography. The difference between the lupus patients with and without PAH in terms of ejection fraction of left ventricle was not statistically significant $(\mathrm{P}>0.05)$, but tricuspid annular plane systolic excursion (TAPSE) and sPAP differed significantly between the two groups of SLE patients $(\mathrm{p}<0.05)$. Pulmonary function tests in all the studied patients revealed restrictive lung disease in 10 patients $(20 \%)$ and normal spirometry in the remaining $(80 \%)$ cases. There was no significant difference between SLE patients with and without PAH with regard to the presence of restrictive and/or obstructive lung diseases ( $>0.05$ ). The $6 \mathrm{MWT}$ was successfully completed by all the patients $(\mathrm{n}=50)$. In this test, mean six-minute walk distance (6MWD) was $480 \mathrm{~m}$ (range: 335-562 m). During this time, oxygen saturation did not occur in any of the patients. At baseline, the dyspnea and fatigue scores were zero, while at the post-exercise stage, mean Borg dyspnea and fatigue scores were 1.37 and 1.49. The difference in total distance walked during six minutes between SLE patients with and without PAH was significant $(p=0.04)$, whereas the pre- and post-exercise Borg dyspnea and fatigue scores were not significantly different between the groups $(p=0.05)$. The mean serum proBNP level measured in this study was $99.64 \mathrm{pg} / \mathrm{ml}$. Comparison of SLE patients with and without PAH showed a significant difference in terms of serum proBNP level $(\mathrm{p}<0.05)$.

Table 2. Comparison of gender distribution in SLE patients with and without PAH

\begin{tabular}{|l|l|l|l|l|}
\hline Parameter & Total $^{\dagger}$ & SLE with PAH $^{\dagger}$ & SLE without PAH $^{\dagger}$ & P value* $^{*}$ \\
\hline Age (year) & $29.10 \pm 5.53$ & $31.4 \pm 4.9$ & $28.8 \pm 5.5$ & 0.267 \\
\hline Gender (F:M) & $48: 2(96: 4)$ & $5: 0(100: 0)$ & $43: 2(95.5: 4.5)$ & 0.512 \\
\hline BMI(kg/m $\left.{ }^{2}\right)$ & $23.46 \pm 3.2$ & $23.7 \pm 2.8$ & $23.43 \pm 3.27$ & 0.788 \\
\hline Disease duration (year) & $4.14 \pm 2.80$ & $2.9 \pm 4.2$ & $1.4 \pm 3.2$ & 0.488 \\
\hline Smoking Hx & 0 & 0 & 0 & ---- \\
\hline Immunosuppressive Tx & $12(24)$ & $2(40.0)$ & $10(22.2)$ & 0.58 \\
\hline Thrombus & $1(2)$ & $0(0.0)$ & $1(2.2)$ & 1.00 \\
\hline Raynaud's & $15(30)$ & $4(80.0)$ & $11(24.4)$ & 0.24 \\
\hline Rheumati Factor & $7(14)$ & $3(60.0)$ & $4(8.9)$ & 0.16 \\
\hline Anti RNP & $17(34)$ & $4(80.0)$ & $13(28.9)$ & 0.40 \\
\hline APL Ab & $11(22)$ & $3(60.0)$ & $8(17.8)$ & 0.64 \\
\hline FEV $(\%$ predict) & $80.78 \pm 9.3$ & $83.69 \pm 8.63$ & $80.45 \pm 9.47$ & 0.28 \\
\hline FEV (L) & $2.76 \pm 0.46$ & $2.77 \pm 0.47$ & $2.63 \pm 0.38$ & 0.48 \\
\hline FVC (\% predict) & $82.9 \pm 10.2$ & $82.54 \pm 10.46$ & $86.40 \pm 7.90$ & 0.29 \\
\hline FVC (L) & $3.13 \pm 0.52$ & $3.14 \pm 0.54$ & $3.09 \pm 0.37$ & 1.00 \\
\hline FEV1/FVC & $88.15 \pm 4.5$ & $88.48 \pm 4.48$ & $85.12 \pm 3.88$ & 0.75 \\
\hline LVEF & $57.1 \pm 3.7$ & $57.11 \pm 3.92$ & $57.0 \pm 2.73$ & 0.951 \\
\hline TAPSE(mm) & $22.1 \pm 3.1$ & $18.40 \pm 2.88$ & $22.51 \pm 2.87$ & 0.007 \\
\hline SPAP(mmHg) & $24.3 \pm 5.4$ & $37.60 \pm 2.51$ & $22.91 \pm 3.21$ & 0.000 \\
\hline MWD (m) 6 & $480.6 \pm 49.9$ & $443.20 \pm 34.96$ & $484.76 \pm 49.95$ & 0.04 \\
\hline Borg dyspnea scale BT. & 0 & 0.00 & 0.00 & --- \\
\hline Borg dyspnea scale AT. & $1.37 \pm 0.72$ & $2.00 \pm 0.70$ & $1.30 \pm 0.69$ & 0.05 \\
\hline Borg fatigue scale BT. & 0 & 0.00 & 0.00 & --- \\
\hline Borg fatigue scale AT. & $1.49 \pm 0.84$ & $2.20 \pm 0.83$ & $1.41 \pm 0.81$ & 0.05 \\
\hline pro BNP level (pg/ml) & $99.6 \pm 87.8$ & $347.00 \pm 34.56$ & $72.16 \pm 27.46$ & 0.000 \\
\hline
\end{tabular}

$\dagger$ Values are expressed as mean \pm standard deviation or counts and percentages; ${ }^{*} \mathrm{p}$ value calculated by Mann Whitney U test / Fisher's exact test 2 sided for comparison between groups of SLE patients with and without PAH. 


\section{Discussion}

In this study, we found that the prevalence rate of PAH in SLE patients was 10\%, which is within the median range of prevalence rates reported from previous studies worldwide (11). All five of the patients diagnosed with PAH in our study had sPAP $<40 \mathrm{mmHg}$, suggesting that $\mathrm{PAH}$ in this study was predominantly mild with no symptoms. The role of left heart disease as a contributing factor in PAH is negligible in the SLE patients participating in our study as none of them had significant left ventricular dysfunction and valvular disease as evaluated by echocardiography. SLE patients with PAH had a shorter mean disease duration (3.2 \pm 1.48 years) in comparison with SLE patients without PAH (4.24 \pm 2.90 years), but this difference was not statistically significant. Our results are congruent with those of another study by Shereen R. Kamel et al., who compared the demographic and clinical features of SLE patients with and without PAH (21). The frequency of Raynaud's phenomenon in both groups of SLE patients showed no significant differences. In a comprehensive literature review conducted in China on seven former studies, $51.2 \%$ of lupus patients with PH had Raynaud's phenomenon contrary to $19.9 \%$ of lupus patients (22). Those studies also reported the correlation of Raynaud's phenomenon with PAH (22). In contrast, in a large cohort of SLE patients in the UK, the association of Raynaud's phenomenon with PAH in SLE patients was not considered statistically significant (11). There was no significant difference between the two groups of patients with regard to antiphospholipid antibody positivity, which was also true for rheumatic factor (RF) and antiU1RNP positivity. A recent cohort study on 200 lupus patients from a tertiary care center did not demonstrate any association between PAH prevalence of $17.5 \%$ and the level of anti-phospholipid antibodies (23), but in another cohort of 288 SLE patients, with PAH prevalence of $4.2 \%$, a statistically significant association between antiphospholipid antibody and PAH was noted (11). In the present study, most of the patients presented with normal spirometry, where abnormal pattern was only noted in $20 \%$ of the patients. All the patients with abnormal spirometry showed restrictive pattern. None of the spirometric parameters were significantly different between the patients with and without PAH and had no value as a screening test for PAH in our study, which is in agreement with results of most previous studies (11). The difference between the total distance walked in the two groups was considered significant; however, no significant correlation was found between the distance walked in 6MWT and sPAP in SLE patients in this study, which is in line with the results of a large cohort study of 283 SLE patients in the UK, in which 6MWT could not differentiate between patients with or without PAH (11). The mean serum proBNP level of the lupus patients with high sPAP was considerably higher at $347 \mathrm{pg} / \mathrm{ml}$ in comparison to $72.16 \mathrm{pg} / \mathrm{ml}$ in the group with normal sPAP $(\mathrm{p}<0.05)$. This is in contrast with the results of another large cohort study conducted by Prabu et al., who found no statistical difference between the proBNP levels in lupus patients with and without PAH (11). Moreover, there was a significant correlation between serum proBNP level and PAP in the current study, and a weak indirect correlation was noted between serum proBNP level and distance walked during six minutes. Nevertheless, Prabu et al. proposed that proBNP level was significantly associated with six minute walk distance, but it was weakly linked with sPAP (11).

\section{Limitations of the study}

There were some limitations to our study as many eligible patients refused to participate due to various reasons including active disease, distance required to travel to the center, work commitments, inconvenience, and lack of interest in participating in the study, which might have biased our results, and in turn, undermined the point prevalence rate of PAH in our study. Our measurements of PAP were indirect and based on screening criteria of echocardiography and were not confirmed by the gold standard RHC measurement of mean PAP, as we considered it unethical to catheterize patients with only mild disease due to its invasive nature. Limited sample size and lack of a control group due to paucity of time and financial resources were other limitations of our study. The overall mild PAH in the current study suggested lack of association between diverse risk factors and screening tests for PAH related to SLE.

\section{Conclusions}

It seems there was no association between PAH and history of thrombosis, Raynaud's phenomenon, or autoantibody positivity in lupus patients. The association of smoking, as a pulmonary risk factor, with PAH in SLE patients remains unclear in our study since none of the patients participating in this study had history of smoking. Considering the PFT results, it indicated to us that subclinical pulmonary involvement is not rare in lupus patients, but spirometric parameters did not show any differences between the groups and may not have screening potential for PAH associated with SLE. The 6MWT distance exhibited no significant correlation with PAP, but showed a weak negative correlation with serum proBNP level in SLE patients. Serum proBNP level was significantly correlated with PAP in patients with SLE. To confirm these results, larger cohort studies on SLE-PAH patients with 
more severe PAH disease should be conducted. We recommend regular evaluation of patients with SLE, regardless of disease duration, for the development of PAH.

\section{Acknowledgments:}

We wish to thank all the patients for their kind cooperation. We would like to thank Mashhad University of Medical Sciences, Mashhad, Iran for their support in this study.

\section{Conflict of Interest:}

There is no conflict of interest to be declared.

\section{Authors' contributions:}

All authors contributed to this project and article equally. All authors read and approved the final manuscript.

\section{References:}

1) Firestein GS, Budd R, Gabriel SE, O'Dell JR, McInnes IB. Kelley's textbook of rheumatology. New York: Elsevier Health Sciences; 2012.

2) Cervera R, Khamashta MA, Font J, Sebastiani GD, Gil A, Lavilla P, et al. Morbidity and mortality in systemic lupus erythematosus during a 10-year period: a comparison of early and late manifestations in a cohort of 1,000 patients. Medicine. 2003; 82(5): 299-308. doi: 10.1097/01.md.0000091181.93122.55. PMID: 14530779.

3) Bernatsky S, Boivin JF, Joseph L, Manzi S, Ginzler E, Gladman D, et al. Mortality in systemic lupus erythematosus. Arthritis Rheum. 2006; 54(8): 2550-7. doi: 10.1002/art.21955. PMID: 16868977.

4) Rubin LJ, Badesch DB, Barst RJ, Galiè N, Black CM, Keogh A, et al. Bosentan therapy for pulmonary arterial hypertension. N Engl J Med. 2002; 346(12): 896-903. doi: 10.1056/NEJMoa012212. PMID: 11907289.

5) Denton CP, Humbert M, Rubin L, Black CM. Bosentan treatment for pulmonary arterial hypertension related to connective tissue disease: a subgroup analysis of the pivotal clinical trials and their open-label extensions. Ann Rheum Dis. 2006; 65(10): 1336-40. doi: 10.1136/ard.2005.048967. PMID: 16793845, PMCID: PMC1798307.

6) Mirfeizi SZ, Lari S, Sahebari M, Attaran D, Pourzand H, Rafatpanah H, et al. The Relationship between Serum Pro - Brain Natriuretic Peptide (Pro - BNP) Levels and Pulmonary Arterial Hypertension (PAH) in Patients with Limited Scleroderma. J Card Thorac Med. 2014; 2(3): 181-6.

7) Simonson J, Schiller N, Petri M, Hellmann D. Pulmonary hypertension in systemic lupus erythematosus. J Rheumatol. 1989; 16(7): 918-25. PMID: 2769664.

8) Johnson S, Gladman D, Urowitz M, Ibanez D, Granton J. Pulmonary hypertension in systemic lupus. Lupus. 2004; 13(7): 506-9. doi: 10.1191/0961203303lu1051oa. PMID: 15352421.

9) Quismorio FP, Sharma O, Koss M, Boylen T, Edmiston AW, Thornton PJ, et al. Immunopathologic and clinical studies in pulmonary hypertension associated with systemic lupus erythematosus. Semin Arthritis Rheum. 1984; 13(4): 349-59. PMID: 6374900.

10) Winslow TM, Ossipov MA, Fazio GP, Simonson JS, Redberg RF, Schiller NB. Five-year follow-up study of the prevalence and progression of pulmonary hypertension in systemic lupus erythematosus. Am Heart J. 1995; 129(3): 510-5. doi: 10.1016/0002-8703(95)90278-3. PMID: 7872181.

11) Prabu A, Patel K, Yee CS, Nightingale P, Situnayake RD, Thickett DR, et al. Prevalence and risk factors for pulmonary arterial hypertension in patients with lupus. Rheumatology. 2009; 48(12): 1506-11. doi: 10.1093/rheumatology/kep203. PMID: 19671698.

12) Rich S. Pulmonary hypertension. In: Bonow R, Mann D, Zipes D, Libby P, editors. Braunwald's heart disease. 9 ed. Philadelphia: Saunders; 2012. p. 1696-716.

13) Mukerjee D, George DS, Knight C, Davar J, Wells A, Du Bois R, et al. Echocardiography and pulmonary function as screening tests for pulmonary arterial hypertension in systemic sclerosis. Rheumatology. 2004; 43(4): 461-6. doi: 10.1093/rheumatology/keh067. PMID: 15024134.

14) Denton C, Cailes J, Phillips G, Wells A, Black C, Bois Rd. Comparison of Doppler echocardiography and right heart catheterization to assess pulmonary hypertension in systemic sclerosis. Br J Rheumatol. 1997; 36(2): 239-43. doi: 10.1093/rheumatology/36.2.239. PMID: 9133938.

15) Tan EM, Cohen AS, Fries JF, Masi AT, Mcshane DJ, Rothfield NF, et al. The 1982 revised criteria for the classification of systemic lupus erythematosus. Arthritis Rheum. 1982; 25(11): 1271-7. doi: 10.1002/art.1780251101. PMID: 7138600. 
16) Golshan M, Nematbakhsh M, Amra B, Crapo R. Spirometric reference values in a large Middle Eastern population. Eur Respir J. 2003; 22(3): 529-34. doi: 10.1183/09031936.03.00003603. PMID: 14516147.

17) Gardner RM, Hankinson JL, West BJ. Evaluating Commercially Available Spirometers. Am Rev Respir Dis. 1980; 121(1): 73-82. doi: 10.1164/arrd.1980.121.1.73. PMID: 7352715.

18) Standardization of spirometry--1987 update. Statement of the American Thoracic Society. Am Rev Respir Dis. 1987; 136(5): 1285-98. doi: 10.1164/ajrccm/136.5.1285. PMID: 3674589.

19) ATS Committee on Proficiency Standards for Clinical Pulmonary Function Laboratories. ATS statement: guidelines for the six-minute walk test. Am J Respir Criti Care Med. 2002; 166(1): 111. doi: 10.1164/ajrccm.166.1.at1102. PMID: 12091180.

20) Borg GA. Psychophysical bases of perceived exertion. Med Sci Sports Exerc. 1982; 14(5): 377-81. doi: 10.1249/00005768-198205000-00012. PMID: 7154893.

21) Kamel SR, Omar GM, Darwish AF, Asklany HT, Ellabban AS. Asymptomatic pulmonary hypertension in systemic lupus erythematosus. Clin Med Insights Arthritis Musculoskeletal Disord. 2011; 4: 77-86. doi: 10.4137/CMAMD.S7667. PMID: 22084605, PMCID: PMC3201107.

22) Xia Y, Tu S, Hu Y, Wang Y, Chen Z, Day H, et al. Pulmonary hypertension in systemic lupus erythematosus: a systematic review and analysis of 642 cases in Chinese population. Rheumatol Int. 2013; 33(5): 1211-7. doi: 10.1007/s00296-012-2525-y. PMID: 22983159, PMCID: PMC3632720.

23) Farzaneh - Far A, Roman MJ, Lockshin MD, Devereux RB, Paget SA, Crow MK, et al. Relationship of antiphospholipid antibodies to cardiovascular manifestations of systemic lupus erythematosus. Arthritis Rheum. 2006; 54(12): 3918-25. doi: 10.1002/art.22265. PMID: 17133599. 